\title{
DNA polymerase hybrids derived from the family-B enzymes of Pyrococcus furiosus and Thermococcus kodakarensis: improving performance in the polymerase chain reaction
}

\author{
Ashraf M. Elshawadfy ${ }^{\dagger}$, Brian J. Keith, $\mathrm{H}^{\prime} \mathrm{Ng}$ Ee Ooi, Thomas Kinsman, Pauline Heslop and \\ Bernard A. Connolly*
}

Institute of Cell and Molecular Biosciences, University of Newcastle, Newcastle upon Tyne, UK

\section{Edited by:}

Zvi Kelman, University of Maryland, USA

\section{Reviewed by:}

Juergen Reichardt, James Cook University, Australia

David L. Bernick, University of

California, Santa Cruz, USA

\section{*Correspondence:}

Bernard A. Connolly, Institute of Cell and Molecular Biosciences (ICaMB), University of Newcastle, The Medical School, Framlington Place, Newcastle upon Tyne, NE24HH, UK e-mail: bernard.connolly@ncl.ac.uk

${ }^{\dagger}$ Present address:

Ashraf M. Elshawadfy, Botany

Department, Zagazig University,

Zagazig, Egypt
The polymerase chain reaction (PCR) is widely applied across the biosciences, with archaeal Family-B DNA polymerases being preferred, due to their high thermostability and fidelity. The enzyme from Pyrococcus furiosus (Pfu-Pol) is more frequently used than the similar protein from Thermococcus kodakarensis (Tkod-Pol), despite the latter having better PCR performance. Here the two polymerases have been comprehensively compared, confirming that Tkod-Pol: (1) extends primer-templates more rapidly; (2) has higher processivity; (3) demonstrates superior performance in normal and real time PCR. However, Tkod-Pol is less thermostable than Pfu-Pol and both enzymes have equal fidelities. To understand the favorable properties of Tkod-Pol, hybrid proteins have been prepared. Single, double and triple mutations were used to site arginines, present at the "forked-point" (the junction of the exonuclease and polymerase channels) of Tkod-Pol, at the corresponding locations in Pfu-Pol, slightly improving PCR performance. The Pfu-Pol thumb domain, responsible for double-stranded DNA binding, has been entirely replaced with that from Tkod-Pol, again giving better PCR properties. Combining the "forked-point" and thumb swap mutations resulted in a marked increase in PCR capability, maintenance of high fidelity and retention of the superior thermostability associated with Pfu-Pol. However, even the arginine/thumb swap mutant falls short of Tkod-Pol in PCR, suggesting further improvement within the Pfu-Pol framework is attainable. The significance of this work is the observation that improvements in PCR performance are easily attainable by blending elements from closely related archaeal polymerases, an approach that may, in future, be extended by using more polymerases from these organisms.

Keywords: PCR, Pyrococcus furiosus, Thermococcus kodakarensis, archaeal DNA polymerase, domain swapping, thermostability, fidelity

\section{INTRODUCTION}

Thermostable DNA polymerases are essential components of the polymerase chain reaction (PCR), a technique with myriad uses across the life sciences (Mullis et al., 1994; Weissensteiner et al., 2004; McPherson and Möller, 2006; Saunders and Lee, 2013). The family-B DNA polymerases from the Thermococcales order of the archaea are especially favored for PCR, due to their extreme stability at elevated temperatures and the presence of fidelity-conferring $3^{\prime}-5^{\prime}$ exonuclease activity (Lundberg et al., 1991; Cline et al., 1996; Takagi et al., 1997; Nishioka et al., 2001). Both the amino acid sequences and X-ray structures of these polymerases demonstrate a high degree of similarity, with structures available for the enzymes isolated from Thermococcus gorgonarius (Tgo-Pol) (Hopfner et al., 1999; Firbank et al., 2008; Killelea et al., 2010), Thermococcus kodakarensis (Tkod-Pol) (Hashimoto et al., 2001; Kuroita et al., 2005; Bergen et al., 2013), Thermococcus species $9^{\circ} \mathrm{N}-7\left(9^{\circ} \mathrm{N}-\mathrm{Pol}\right)$ (Chapin-Rodriguez et al., 2000), Pyrococcus furiosus (Pfu-Pol) (Kim et al., 2008) and
Pyrococcus abysii (Pab-Pol) (Gougel et al., 2012). Despite similarities of amino acid sequence and structure, these polymerases have diverse kinetic properties; a strong influence on PCR performance. Most notably Tkod-Pol possesses higher processivity (the number of dNTPs incorporated per binding event) than other enzymes (Takagi et al., 1997; Nishioka et al., 2001). Enhanced processivity may arise from the presence of seven arginines, suggested to play a role in stabilizing primer-template binding and influencing the movement of DNA between the polymerization and proof reading active sites (Hashimoto et al., 2001; Kim et al., 2008). These arginines cluster near the "forked-point" (the junction between the template-binding and editing clefts) of Tkod-Pol (Figure 1A). The seven amino acids are well conserved in Thermococcales DNA polymerases, with two (R243 and R264) being present in all species (Figure 1B). The other five locations show more variation, although the amino acid at 266 is an arginine in both Tkod-Pol and Pfu-Pol. At the remaining four positions $(247,365,381,501)$, the arginines present in Tkod-Pol 


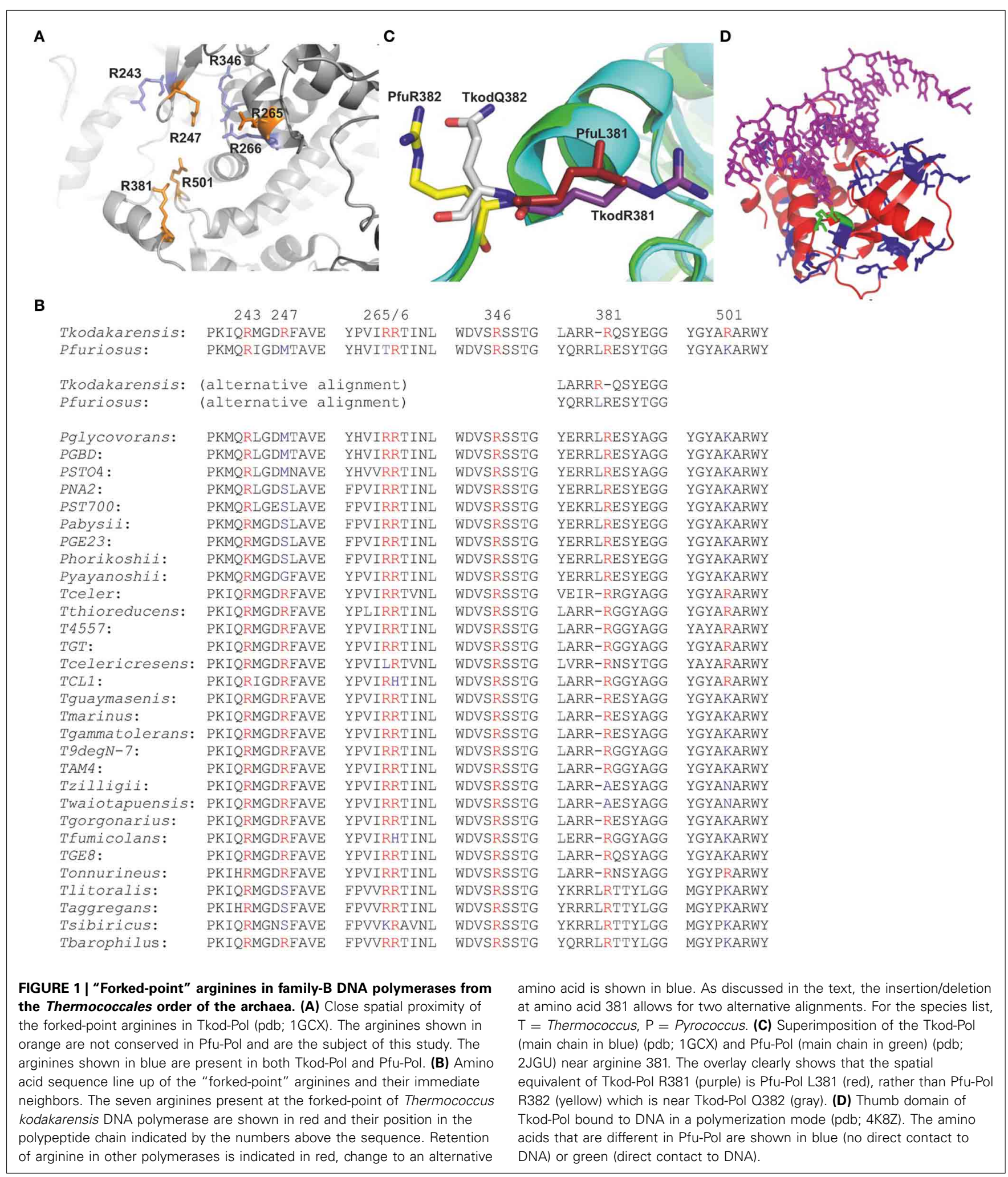

are replaced by an alternative amino acid. The situation at TkodPol position 381 is slightly obscured by insertion of an additional leucine at position 381 in Pfu-Pol. This arrangement, seen with many of the Thermococcales DNA polymerases, leads to two possible sequence alignments (Figure 1B). The first, generated by the alignment algorithm Clustal (Sievers et al., 2011), lines up Tkod-Pol R381 with Pfu-Pol R382. However, superimposition of the structures of the two polymerases (Figure 1C) shows 
that R381 in Tkod-Pol and L381 in Pfu-Pol are spatially equivalent, suggesting the alternative alignment shown in Figure 1B (Hashimoto et al., 2001). Another region that may contribute to processivity is the thumb domain, responsible for binding double-stranded DNA (Firbank et al., 2008; Killelea et al., 2010; Gougel et al., 2012; Bergen et al., 2013). This domain grips DNA tightly and interacts with newly synthesized double-strands, implying an important role in DNA translocation. In the absence of DNA, the thumb shows high flexibility and stretches of amino acids are often invisible in apo-enzyme crystal structures. The overall fold of the thumb domain does not differ significantly between archaeal DNA polymerases; however, its location relative to other domains is quite variable and entire domain motion is observed on DNA binding. Although the amino acid sequences in this domain are similar overall, many changes to individual amino acids are seen when Pfu-Pol and Tkod-Pol are compared (Figure 1D; supplementary data, Figure S1). However, in the current absence of polymerase-DNA-dNTP ternary structures, it is not easy to correlate any differences in processivity between PfuPol and Tkod-Pol with individual amino acids in the thumb region.

During archaeal replication processivity is facilitated by PCNA, a sliding clamp which completely encircles the DNA, while simultaneously binding to the polymerase (Ishino and Ishino, 2011). Polymerase dissociation from DNA is hindered and overall processivity is enhanced, leading to rapid copying of the genome. In the thermococcales PCNA is a homo-trimeric ring-shaped molecule and the ring must be opened to load the protein onto DNA. In vivo, loading of PCNA is carried out by RFC, an ATPdriven clamp loader (Ishino and Ishino, 2011). However, in vitro, e.g., during the PCR, PCNA is not usually present and copying of DNA relies on the intrinsic processivity of the polymerase. Occasionally PCNA has been added and has been reported to enhance the PCR capability of Tkod-Pol (Kitabayashi et al., 2002). A recent comprehensive study showed that native Pfu-PCNA only improved PCR if RFC was also present, presumably to facilitate loading of the clamp onto DNA (Ishino et al., 2012). However, two PCNA mutants with reduced ring stability where able to self-load and considerably improved the PCR performance of Pfu-Pol, in the absence of RFC. For biotechnology applications more processive polymerases have been generated by fusion with double-stranded DNA binding proteins such as helix-hairpinhelix motifs (Pavlov et al., 2002) and Sso7d (a thermostable protein from Sulfolobus solfataricus) (Wang et al., 2004); the latter giving significant improvement in many PCR applications. Elevated processivity leads to more rapid copying of DNA and a polymerase with this property should be better at the PCR i.e., generate product more rapidly or produce longer amplicons. As processivity, in vivo, is largely a function of the sliding clamp, there may be little evolutionary pressure on polymerases to maximize this function, allowing considerable scope for improvement. In this publication we have sought to rationalize the differences in processivity and PCR ability previously observed between TkodPol and Pfu-Pol. Hybrid polymerases have been produced, mainly Pfu-Pol but containing amino acids/domains from Tkod-Pol suggested to contribute to processivity. Do these hybrids result in a transfer of processivity from Tkod-Pol to Pfu-Pol and improved
PCR performance? A better comprehension of why two structurally similar DNA polymerases have different kinetic properties is intrinsically important and may also guide further rational mutagenesis.

\section{MATERIALS AND METHODS PROTEIN PURIFICATION AND MUTAGENESIS}

Wild type Pfu-Pol B (gene inserted into pET17b) was purified as reported (Evans et al., 2000; Emptage et al., 2008). Wild type TKod-Pol B (present in pET21a, supplied by Dr. Zvi Kelman University of Maryland) was purified in an identical manner. The single, double and triple mutants of Pfu-Pol were produced using a QuickChange ${ }^{\circledR}$ site directed mutagenesis kit (AgilentStratagene, Stockport) with Velocity ${ }^{\mathrm{TM}}$ DNA polymerase (Bioline, London, UK). The genes encoding the Pfu-TKod thumb swap derivatives were prepared using overlap extension PCR (Warrens et al., 1997). All mutated genes were completely sequenced to ensure the presence of the desired mutation and an absence of changes elsewhere. All mutants were purified in the same manner as the wild type enzyme with SDS-PAGE and Coomassie Blue staining indicating a purity of $>95 \%$.

\section{POLYMERASE AND EXONUCLEASE ASSAYS}

Primer-template extensions used the fluorescent-labeled primertemplates given in the legends to Figures 2, 3 and supplementary data Figures S2, S3. These reactions were conducted, at $30^{\circ} \mathrm{C}$, in $400 \mu \mathrm{l}$ of $20 \mathrm{mM}$ Tris $\mathrm{pH} 8.0,10 \mathrm{mM} \mathrm{KCl}, 10 \mathrm{mM}\left(\mathrm{NH}_{4}\right)_{2} \mathrm{SO}_{4}$, $2 \mathrm{mM} \mathrm{MgSO}_{4}, 0.1 \%(\mathrm{v} / \mathrm{v})$ Triton X-100 and $40 \mu \mathrm{g}$ of bovine serum albumin. $400 \mu \mathrm{M}$ of each dNTP was used with $10 \mathrm{nM}$ primer-template and reactions were initiated by adding the polymerase to a final concentration of $500 \mathrm{nM}$. Subsequently $40 \mu \mathrm{l}$ aliquots were sampled at appropriate time-points by quenching with an equal volume of stop buffer (95\% formamide, $10 \mathrm{mM}$ EDTA, $10 \mathrm{mM} \mathrm{NaOH}, 2 \mu \mathrm{M}$ of a competitor oligodeoxynucleotide and $0.05 \%$ xylene cyanol indicator dye. Primer-templates were denatured by heating at $100^{\circ} \mathrm{C}$ for $10 \mathrm{~min}$ followed by cooling on ice. The competitor, which has the same sequence as the fully extended primer but lacks the fluorophore, prevents significant re-hybridization of the fluorescent products to the template (Russell et al., 2009). The samples $(20 \mu \mathrm{l})$ were resolved on a denaturing $17 \%$ polyacrylamide gel containing $8 \mathrm{M}$ urea run at 4.5 Watts for $4.5 \mathrm{~h}$ and visualized using a Typhoon scanner with ImageQuant software (GE Healthcare). Exonuclease assays were performed similarly, save for omission of the dNTPs and data were fitted to a first order reaction (\% substrate remaining $=100 \mathrm{e}^{-\mathrm{kt}}+$ offset; $k=$ rate constant, $t=$ time) using GraFit (Erithacus Software, London, UK), giving rate constants.

\section{REAL TIME PCR}

Real-time PCR used a Rotor-Gene 6000 thermocycler (Corbett Research, Qiagen). Genomic DNA from S. cerevesiae was used as the template, with the DNA Pol 2 gene targeted for amplification. A common forward primer (TACGTACCGCCGCAATACAA TGGCAGG) and four different reverse primers (TCGAATTG CCGCCGCCATTACTACCAC, TCGACTTGAAGCTCCCACC CTCTTCATC, GGCGTCAACTTTTTCCGAGCCATTTGC and TCATCGAACATGTCCAAGCCGTGAATCTTAC) were used to 


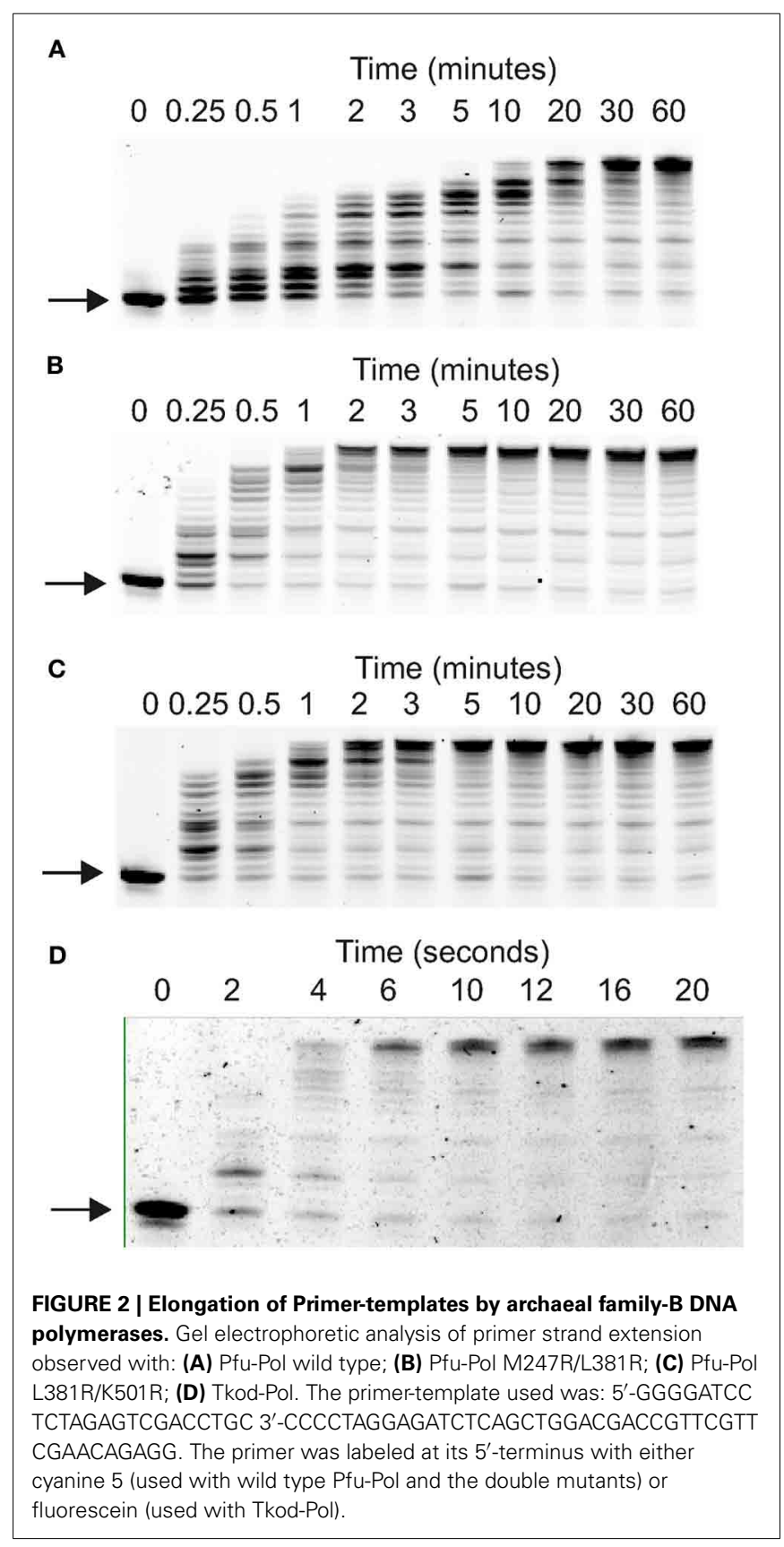

amplify lengths of $145,232,543$, and 1040 base pairs. Reactions were carried out in $25 \mu \mathrm{l}$ that contained $30 \mathrm{ng}$ S. cerevesiae genomic DNA (Novagen), $1 \mu \mathrm{M}$ of each primer, $400 \mu \mathrm{M}$ of each $\mathrm{dNTP}$, and $2.5 \mu \mathrm{l}$ of SYBR green $(10,000 \times$ stock in dimethlysulfoxide, Invitrogen; initially diluted 1000 -fold with water). The reactions were initiated by adding the polymerase (final concentration $20 \mathrm{nM}$ ) in the same buffer used for primer-template extensions. The PCR consisted of $1 \times 95^{\circ} \mathrm{C}$ for $2 \mathrm{~min}$ followed by 40 cycles of: $95^{\circ} \mathrm{C}(10 \mathrm{~s}) / 58^{\circ} \mathrm{C}(20 \mathrm{~s}) / 72^{\circ} \mathrm{C}$ (the time used for the $72^{\circ} \mathrm{C}$ extension step varied as indicated in the results). On completion of the PCR a melt curve analysis, consisting of a $90 \mathrm{~s}$ pre-melt step at $67^{\circ} \mathrm{C}$ followed by a temperature increase to $95^{\circ} \mathrm{C}$ at $0.2^{\circ} \mathrm{C}$ per second, was carried out. $20 \mu l$ of the real time PCR mixtures were run on a $1 \%$ agarose gel (detection with ethidium bromide) in order to verify that amplification resulted in a product of the correct size.

\section{PCR}

Amplification of a stretch of DNA $\sim 5 \mathrm{kbases}$ long within the plasmid pET17b[Pfu-Pol] (Evans et al., 2000) was carried out in $50 \mu \mathrm{l}$ using TCTGCTATGTGGCGCGGTATTATCC and CAACTCAGCTTCCTTTCGGGCTTTG $(1 \mu \mathrm{M}$ each $)$ as primers, $400 \mu \mathrm{M}$ of each of the $4 \mathrm{dNTPs}$ and either 20 or $100 \mathrm{nM}$ of polymerase. $50 \mathrm{ng}$ of pET17b[Pfu-Pol] was used with two buffers, either $20 \mathrm{mM}$ Tris- $\mathrm{HCl}$ pH 8 or $20 \mathrm{mM}$ Bicine- $\mathrm{NaOH}$ pH 9 both containing $10 \mathrm{mM} \mathrm{KCl}, 10 \mathrm{mM}\left(\mathrm{NH}_{4}\right)_{2} \mathrm{SO}_{4}, 2 \mathrm{mM} \mathrm{MgSO}_{4}, 0.1 \%$ (v/v) Triton-X100 and $5 \mu \mathrm{g}$ bovine serum albumin. The PCR cycle comprised: pre-heat at $98^{\circ} \mathrm{C}(2 \mathrm{~min}) ; 30$ cycles of $95^{\circ} \mathrm{C}$ $(30 \mathrm{~s}), 60^{\circ} \mathrm{C}(30 \mathrm{~s}), 70^{\circ} \mathrm{C}(5 \mathrm{~min})$; final hold at $70^{\circ} \mathrm{C}$ for $5 \mathrm{~min}$. $20 \mu \mathrm{l}$ of the PCR mixtures (expected size of the correct amplicon $\sim 5 \mathrm{~kb}$ ) were analyzed using $1 \%$ agarose gel electrophoresis with ethidium bromide staining.

\section{POLYMERASE FIDELITY}

Fidelities were determined using pSJ2, an assay based on gapfilling of the single stranded lac $Z \alpha$ segment within the plasmid by a polymerase in vitro. The gapped derivative of pSJ2 ( $1 \mathrm{nM})$ was fully extended using the polymerase $(100 \mathrm{nM})$ in $20 \mu \mathrm{l}$ of $20 \mathrm{mM}$ Tris $(\mathrm{pH} 8.0), 10 \mathrm{mM} \mathrm{KCl}, 10 \mathrm{mM}\left(\mathrm{NH}_{4}\right)_{2} \mathrm{SO}_{4}, 2 \mathrm{mM}$ $\mathrm{MgCl}_{2}, 0.1 \%$ (v/v) Triton X-100, $2 \mu \mathrm{g}$ bovine serum albumin and $250 \mu \mathrm{M}$ of each of the four dNTPs. Extension was carried out at $70^{\circ} \mathrm{C}$ for $30 \mathrm{~min}$ and the mixture was used to transform E. coli Top 10 cells, which were plated on LB agar (containing X-gal, IPTG and ampicillin) and scored for blue/white colonies. Ratios of blue/white colonies were converted to mutation frequency and error rate as previously described (Jozwiakowski and Connolly, 2009; Keith et al., 2013).

\section{PRIMER-TEMPLATE BINDING}

The binding of the polymerases to primer-templates was determined using fluorescence anisotropy with hexachlorofluoresceinlabeled DNA (Shuttleworth et al., 2004). The buffer conditions are detailed in the supplementary data (Figure S6).

\section{PROCESSIVITY}

Four hundred $\mu \mathrm{l}$ of $20 \mathrm{mM}$ Tris $\mathrm{pH} 8.0,10 \mathrm{mM} \mathrm{KCl}, 10 \mathrm{mM}$ $\left(\mathrm{NH}_{4}\right)_{2} \mathrm{SO}_{4}, 1 \mathrm{mM}$ EDTA, $0.1 \%$ Triton $\mathrm{X}-100,40 \mu \mathrm{g}$ of bovine serum albumin, $400 \mu \mathrm{M}$ of each of the four dNTPs, $40 \mathrm{nM}$ primer-template (sequence given in the legend to Figure 7) and $500 \mathrm{nM}$ of polymerase were pre-incubated at $50^{\circ} \mathrm{C}$ for $5 \mathrm{~min}$. Reactions were initiated by the simultaneous addition of $3 \mathrm{mM}$ $\mathrm{MgSO}_{4}$ and $10 \mu \mathrm{M}$ of a uracil-rich oligodeoxynucleotide trap (sequence given in legend to Figure 7) and polymerization allowed to proceed at $50^{\circ} \mathrm{C}$. Aliquots of $40 \mu \mathrm{l}$ were withdrawn at appropriate times and extension determined using denaturing gel electrophoresis as described above.

\section{DSF ANALYSIS}

The thermal stabilities of the polymerases were analyzed by heating samples in a Rotor-Gene 6000 (Corbett Research, Qiagen) in the presence of SYPRO Orange and measuring any increase 

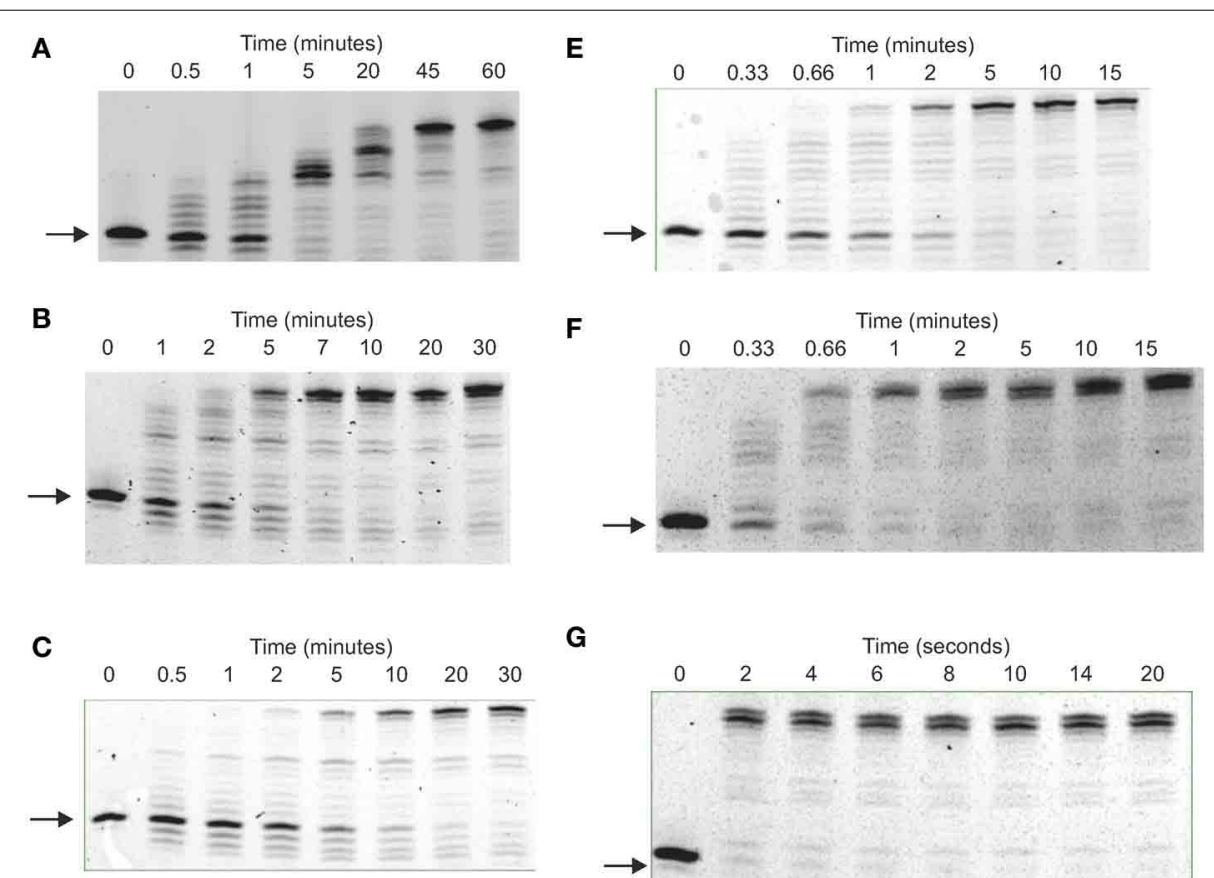

G
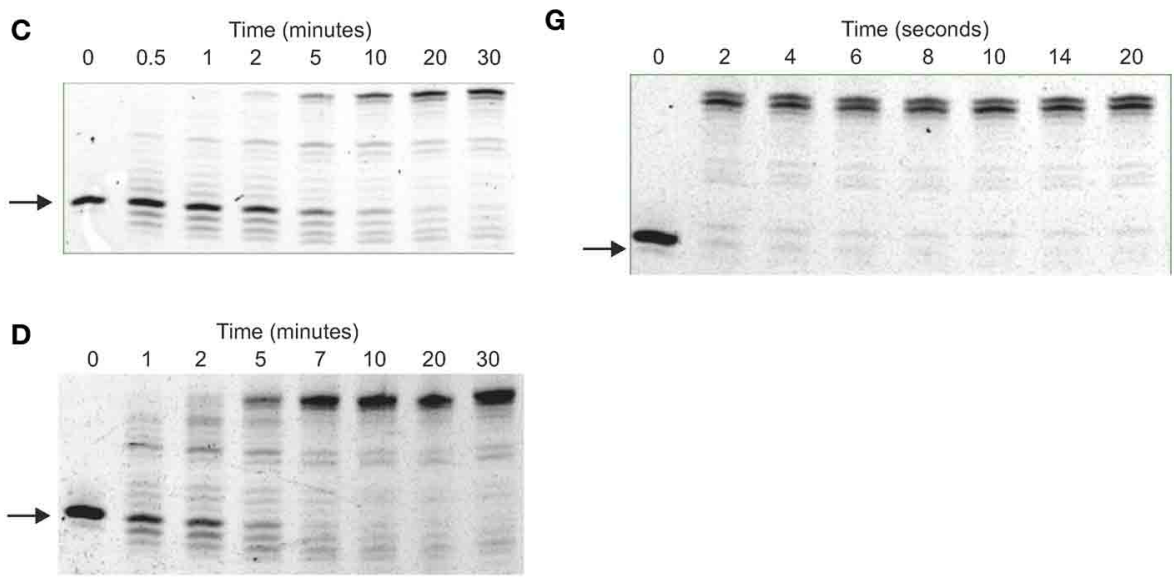

FIGURE 3 | Elongation of primer-templates by archaeal family-B DNA polymerases. Gel electrophoretic analysis of primer strand extension observed with: (A) Pfu-Pol wild type; (B) Pfu-Pol M247/L381; (C) Pfu-Pol L381R/K501R; (D) Pfu-Pol M247R/L381R/K501R; (E) Pfu-TkodTS;

(F) Pfu-TkodTS L381R/K501R; (G) Tkod-Pol wild type. The primer-template used was: 5'-GGGGATCCTCTAGAGTCGACCTGCAGGGCAA 3'-CCCCTAGGA GATCTCAGCTGGACGTCCCGTTCGTTCGAACAGAGG. The primer was labeled at its $5^{\prime}$-terminus with fluorescein.

in fluorescence. Thermal melts were carried out in $100 \mu \mathrm{l}$ of $40 \mathrm{mM}$ HEPES, pH 7, containing $400 \mathrm{mM} \mathrm{NaCl}, 2 \mathrm{M}$ guanidinium hydrochloride, $10 \mu \mathrm{l}$ of SYPRO Orange $(5000 \times$ stock in dimethylsulfoxide, Sigma; initially diluted 100 -fold with water). The polymerases were used at a concentration of $2 \mu \mathrm{M}$. Excitation and emission were at $470 \mathrm{~nm}$ and $555 \mathrm{~nm}$, respectively. The temperature increased from 25 to $100^{\circ} \mathrm{C}$ at a rate of $1^{\circ} \mathrm{C}$ per min. Data analysis was carried out with the Rotor-Gene 6000 series software and the melting profiles are presented as first derivatives.

\section{RESULTS \\ MANIPULATING AMINO ACIDS AND DOMAINS OF PfU-Pol TO MAKE IT MORE SIMILAR TO TKOd-Pol}

TKod-Pol contains seven arginines at the forked-point, four of which are replaced in Pfu-Pol (Figures 1A,B). The absent arginines have been investigated by introducing them into Pfu-Pol, initially as the single amino acid substitutions, PfuPolM247R, T265R, and K502R. The situation at Tkod-Pol position 381 is more complex, due to the insertion of an additional leucine at position 381 in Pfu-Pol (Figure 1B). Therefore, two $\mathrm{Pfu}$-Pol variants have been created, corresponding to the potential sequence line ups shown in Figure 1B. The deletion of L381, to give Pfu-PolL381 $\Delta$, removes the extra amino acid, which may bring R382 in Pfu-Pol into register with R381 in TkodPol. Additionally the direct substitution mutation, Pfu-PolL381R, has been prepared, corresponding more closely to structural data (Figure 1C). Insertion of L381 also means that lysine 502 in Pfu-Pol corresponds to arginine 501 in TKod-Pol. Several of the single amino acid modifications to Pfu-Pol showed improved ability to copy DNA (next section). To generate incremental increases in polymerization activity, two double mutants, PfuPolM247R/L381R and Pfu-PolL381R/K502R and a triple variant, Pfu-PolM247R/L381R/K502R were prepared.

A second Pfu-Pol mutant has the thumb domain (amino acids T591 to S775 at the carboxyl terminus) replaced with the corresponding region from Tkod-Pol (named Pfu-TkodTS, TS = thumb swap). There are multiple differences in the amino acid sequences that comprise the thumb domains of the two polymerases (Figure 1D; supplementary data figure S1). Of the amino acids in the Tkod thumb that contact DNA directly, only two, R709 and G711 (shown in green in Figure 1D), are changed in Pfu-Pol, to proline and serine, respectively. However, many Tkod 
residues, immediately adjacent to a DNA-contacting amino acid, also vary in Pfu-Pol (supplementary data Figure S1). The numerous variations in sequence make it a sizeable task to probe the contribution of individual amino acids; rather a complete thumb transplant was used. Finally the most advantageous "forkedpoint" double mutant (L381R/K502R) has been combined with the thumb domain swap.

\section{PRIMER-TEMPLATE EXTENSION BY DNA POLYMERASES}

Polymerase activities were initially compared using several primer-templates, a simple extension assay useful for indicating the effectiveness with which DNA is copied. The single amino acid substitutions Pfu-Pol M247R and K502R generated fulllength product more rapidly than wild type (supplementary data, Figure S2). At amino acid position 381, where two mutations were made, L381R appeared slightly better than L381 $\Delta$ and both were superior to wild type. Pfu-Pol T265R was the only instance of a substitution giving poorer extension (supplementary data, Figure S3). Building on the single-swaps, two double mutants (M247R/L381R and L381R/K502R) were created; both extended the primer-template significantly more rapidly than wild type and slightly faster than their single parents. Faster extension with the double mutants was consistently observed with three primer-templates (Figures 2, 3; supplementary data Figure S2). As can be seen in Figure 2, wild type Pfu-Pol required 10-20 min before full length product became visible, whereas completely extended primer was apparent after 1-2 min with both of the doubles. An alternative primer-template (Figure 3) again indicated more rapid extension with the double mutants (compare the five and twenty minute time points for wild type, M247R/L381R and L381R/K502R in Figure 3). This figure additionally demonstrates that the triple mutation (M247R/L381R/K502R) shows little additive improvement. Although Pfu-Pol M247R/L381R and L381R/K502R are superior to wild type, they do not match the performance of Tkod-Pol, where full extension is apparent after less than $10 \mathrm{~s}$ (Figures 2, 3). The assay was also shows that PfuTkodTS extends primer-template more rapidly than wild type (Figure 3). With Pfu-TkodTS the reaction is almost complete after $5 \mathrm{~min}$, whereas little full length product is seen with the wild type after this time. Even faster extension was seen when PfuTkodTS/L381R/K502R was investigated, with the reaction finishing in $1-2 \mathrm{~min}$. Extensions were carried out at $30^{\circ} \mathrm{C}$, away from the temperature optima of about $75^{\circ} \mathrm{C}$ for Pfu-Pol and Tkod-Pol (Takagi et al., 1997). Maybe, as temperature decreases, the activity of Pfu-Pol declines more steeply than Tkod-Pol, explaining the observed superior performance of Tkod-Pol at $30^{\circ} \mathrm{C}$. However, Tkod-Pol maintained its advantage in both real time and standard PCR (Figures 4, 5 respectively), techniques that involve DNA synthesis near the temperature optimum. Based on primer-template extensions, four mutants, M247R/L381R, L381R/K502R, PfuTkodTS, and Pfu-TkodTSL381R/K502R were selected for more detailed investigation.

\section{REAL TIME PCR ANALYSIS}

A key motivation in manipulating Pfu-Pol was to produce superior PCR enzymes and, to allow direct identification of useful mutants, priority was given to PCR-based assays. Initially real
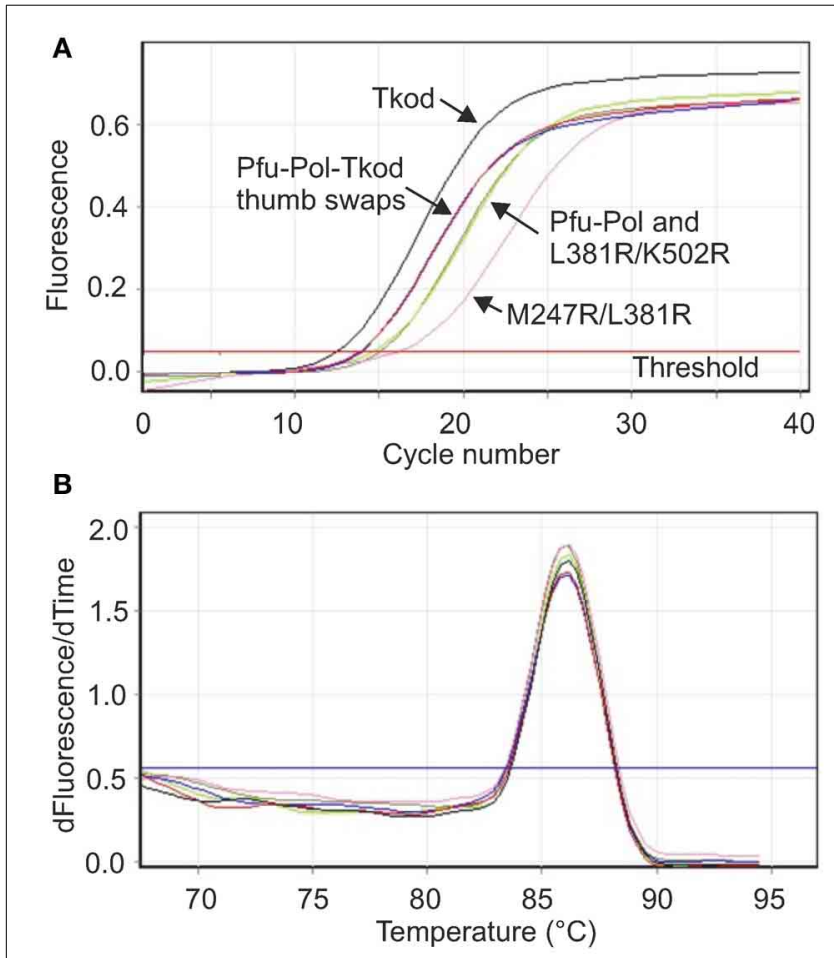

FIGURE 4 | Real time PCR analysis of polymerase performance.

(A) Amplification of a stretch of yeast genomic DNA 232 bases in length using $10 \mathrm{~s}$ extension. The lines that correspond to the individual polymerases are identified on the figure. Pfu-Pol TS and Pfu-Pol TS L381R/K502R (indicated Pfu-Tkod thumb swaps) gave near superimposable lines. Likewise the lines for Pfu-Pol and the double mutant L381R/K502R overlapped strongly. (B) Melting temperature analysis (first derivative showing the rate of change of temperature with time against time) of the amplicons generated in (A). All the polymerases gave exclusively the desired product as indicated by a single peak with a $T_{m}$ of $86^{\circ} \mathrm{C}$. As all the lines are essentially identical the individual polymerases have not been identified. In both panels only a single line for each polymerase is shown but all experiments were carried out in triplicate.

time PCR (RT-PCR), commonly used to measure DNA and RNA levels (Saunders and Lee, 2013), was applied to investigate polymerase performance. During RT-PCR a $C_{t}$ value defines the number of cycles taken for product to become apparent; the more effective the polymerase, therefore, the lower the $C_{t}$. In these experiments, yeast genome DNA targets of 232, 543, and 1040 bases were generated and the products detected using the fluorophore SYBR green. With the shortest amplicon (232 bases), Pfu-Pol and the double mutant L381R/K502R generated product after a similar number of cycles $\left(C_{t} 15.30\right.$ and 14.93, respectively) and M247R/L381R was slightly slower $\left(C_{t}\right.$ 15.96). Here, the behavior of M247R/1381R is anomalous as it appeared superior to the wild type in every other test of polymerization ability. Tkod-Pol produced product more rapidly than the wild type i.e., had a lower $C_{t}$, and the two thumb swap mutants showed intermediate $C_{t}$ values. As an example the real time PCR data found with the short amplicon is shown in Figure 4 and all $C_{t}$ values are summarized in Table 1. Further information was obtained using a longer amplicon of 543 bases and varying the extension time. 


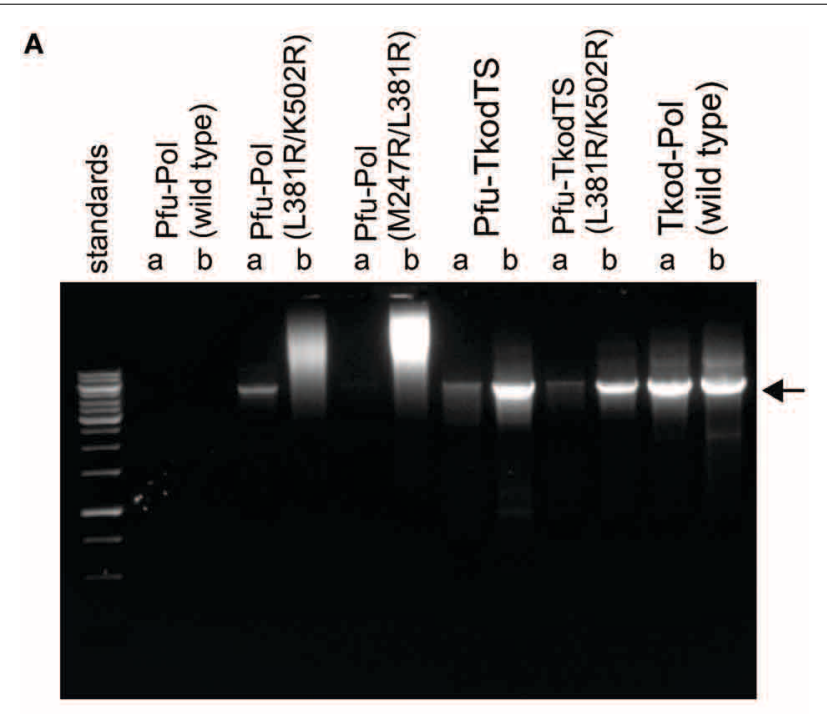

B

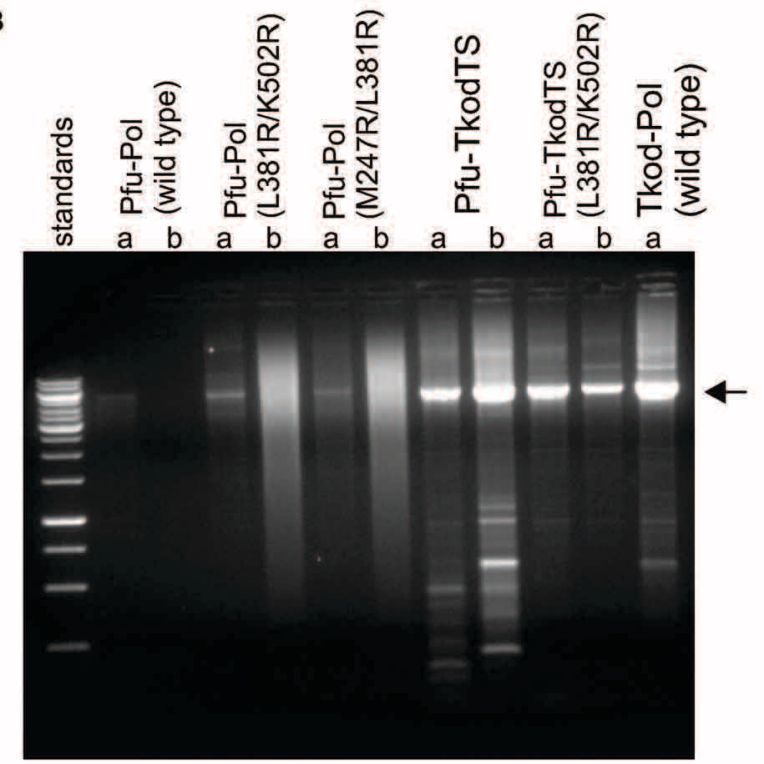

FIGURE 5 | PCR amplification of $\sim 5 \mathrm{~kb}$ of DNA in pET17b[Pfu-Pol] by polymerase variants. Reactions were carried out using the polymerase variants indicated $(a=20 \mathrm{nM} ; b=100 \mathrm{nM})$ in two buffer systems $(\mathbf{A}=$ Tris- $\mathrm{HCl} \mathrm{pH} 8 ; \mathbf{B}=$ Bicine- $\mathrm{NaOH}$ pH 9). Analysis was by $1 \%$ agarose gel electrophoresis with ethidium bromide staining. The standards comprise a $1 \mathrm{~kb}$ ladder with the more intense bands at 1,3 , and $6 \mathrm{kB}$. The expected PCR product $(\sim 5 \mathrm{~kb})$ is indicated with the arrow.

The double mutants appeared superior to Pfu-Pol, either giving a product where none was produced by the parent or generating amplicons more quickly; L381R/K502R demonstrated better performance than M247A/L38R. Greater efficiency was again apparent for Tkod-Pol, which generated products more rapidly as measured by $C_{t}$ values. Similarly the thumb swap mutants showed better performance than Pfu-Pol (wild type and the double mutants) but were inferior to Tkod-Pol (Table 1). Confirmation of these trends came from the use of the longest amplicon (1040 bases) with four different extension times. Overall, as summarized in Table 1, Tkod-Pol gives the best performance in RT-PCR followed, in order, by Pfu-TkodTSL381R/K502R, Pfu-TkodTS, Pfu-PolL381R/K502R, Pfu-PolM247R/L381R, with Pfu-Pol wild type, the worst enzyme. This ranking is an excellent match to the results seen in primer-template extensions. While RT-PCR is a straightforward method for characterizing polymerases, incorrect products are often produced. Therefore, the integrities of the 232, 543, and 1040 base products were checked using melting temperature analysis (all three had similar $T_{m}$ values of between 86 and $88^{\circ} \mathrm{C}$; for an example see Figure 4 ) and by gel electrophoresis to confirm the presence of product with the expected length (supplementary data, Figure S4). In Table 1, $C_{t}$ values are only quote if the anticipated product comprised at least $95 \%$ of the amplification mixture.

\section{PCR PERFORMANCE OF POLYMERASES}

To determine if the more rapid primer-template extensions and lower $C_{t}$ values exhibited by the Pfu-Pol mutants translated into improved standard PCR performance, amplifications of a $5 \mathrm{~kb}$ stretch of DNA were carried out. Two buffers with $\mathrm{pH}$ of 8 and 9 were used and the polymerases were tested at two concentrations, 20 or $100 \mathrm{nM}$. The results are given in Figure 5 and show that wild type Pfu-Pol failed to generate substantial amounts of product, traces being apparent only at $\mathrm{pH} 9$ with $20 \mathrm{nM}$ enzyme. The double mutants M247A/L381R were better; at $20 \mathrm{nM}$ levels both gave obvious product at $\mathrm{pH} 9, \mathrm{~L} 381 \mathrm{R} / \mathrm{K} 502 \mathrm{R}$ also yielded product at $\mathrm{pH} 8$. However, at the higher concentration of $100 \mathrm{nM}$ the doubles gave smeared bands, suggesting non-specific amplification. The thumb swaps showed marked PCR improvement, at both $\mathrm{pH}$ values and protein concentrations a clear and intense (apart from $\mathrm{pH} 8,20 \mathrm{nM}$ ) product band was visible, suggesting efficient amplification. Some non-specific products were apparent, particularly at $\mathrm{pH}$ 9. Wild type Tkod-Pol also performed well, perhaps marginally better than the two thumb swap mutants. Non-specific smaller products were also observed with Tkod-Pol at $\mathrm{pH}$ 9. The PCR results concur with all those above; the double mutants are superior to wild type Pfu-Pol and the thumb swaps even better. In these experiments the thumb swaps approach the performance exhibited by wild type Tkod-Pol.

\section{FIDELITY OF DNA POLYMERASES}

DNA polymerase accuracy is crucial for PCR and the main reason archaeal enzymes are widely applied. Two techniques were used to check the fidelity of the enzymes. Initially proof reading exonuclease activity was directly measured using a primer-template containing a base mismatch at the point of extension. A protein concentration $(500 \mathrm{nM})$ in excess of primer-template $(10 \mathrm{nM})$ was used and exonuclease activity observed by monitoring the removal of the mismatched base at the $3^{\prime}$-end of the primer. All Pfu-Pol mutants demonstrated slightly faster exonuclease rate constants than wild type, although increases were at most a factor of two (Figure 6, Table 2). Noticeably wild type Tkod-Pol showed more pronounced activity, about 7.5 times faster than Pfu-Pol. The plots shown in Figure 6 and the rate constants in Table 2 measure the disappearance of initial primer and so report on removal of the mismatched base. It is abundantly clear that subsequent degradation of the primer can occur, as a consequence of exonuclease activity at Watson-Crick base pairs. With Tkod-Pol 
Table 1 | RT-PCR performance of the polymerase variants.

\begin{tabular}{|c|c|c|c|c|c|c|c|}
\hline \multicolumn{2}{|c|}{ Experimental conditions } & \multicolumn{6}{|c|}{$C_{t}$ value of polymerase variant ${ }^{b}$} \\
\hline 543 & 10 & NP & NP & NP & 14.65 & 12.73 & 10.94 \\
\hline 543 & 30 & NP & 12.20 & 10.55 & 8.61 & 8.07 & 6.72 \\
\hline 1040 & 10 & NPc & NP & NP & NP & NP & 12.29 \\
\hline 1040 & 30 & NP & NP & NP & NP & 16.90 & 11.05 \\
\hline 1040 & 60 & NP & NP & 16.37 & 14.51 & 12.74 & 10.83 \\
\hline 1040 & 90 & NP & NP & 13.85 & 12.66 & 11.10 & 10.75 \\
\hline
\end{tabular}

a Yeast genome DNA was the target for amplification and primers (given in Materials and Methods) were selected to give the amplicon lengths indicated.

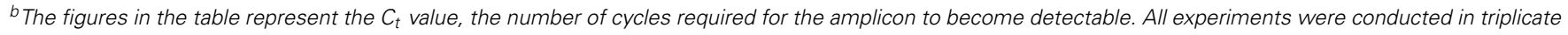

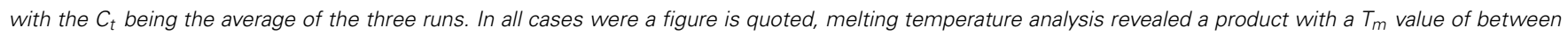

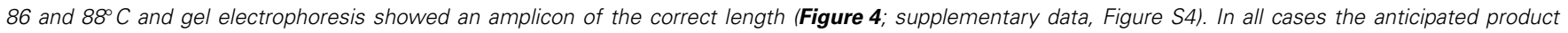
comprised at least $95 \%$ of the total amplified material.

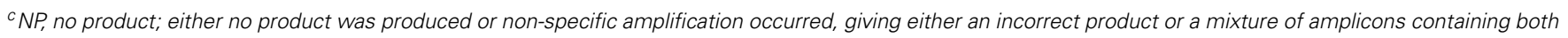
the desired and non-specific products.

accumulation of the n-1 product is clear, suggesting preferential degradation of mismatched bases. Equivalent accumulation is less obvious with Pfu-Pol, inferring less discrimination between mismatches and bona fide base-pairs in this case. To further investigate the proof reading activities of Pfu-Pol and Tkod-Pol were compared using a fully complementary primer-template. With these undamaged substrates the exonuclease activity of Tkod-Pol was only 1.5 fold faster than Pfu-Pol, less than the factor observed with the mismatched substrate (supplementary data, Figure S5). The faster exonuclease rate of Tkod-Pol, compared to Pfu-Pol, with mismatches, followed by near equal reactivity at normal bases accounts for the n-1 product accumulation seen in Figure 6 . Exonuclease activities were greater with mismatched than fully complementary substrates (Table 1); an expected observation as mismatches are easier to unwind, a necessary step for proof reading (Reha-Krantz, 2010).

To complement the measurements of exonuclease activities, fidelities have been determined using a lac $Z \alpha$ plasmid-based assay (Jozwiakowski and Connolly, 2009; Keith et al., 2013). A gapped plasmid, which contains the lac $Z \alpha$ gene in the single stranded region, is fully extended, in vitro at $70^{\circ} \mathrm{C}$, by a polymerase and then used for transformation of an appropriate E. coli strain. The observed ratio of white to blue colonies on plates contain the indicator X-gal, reflects the fidelity of the polymerase. For these experiments pSJ2 was used, a plasmid that has been characterized sufficiently to allow conversion of white/blue ratio i.e., the mutation frequency into error rate, the number of mistakes made per base incorporated (Keith et al., 2013). The results observed are given in Table 3, which demonstrates near identical error rates for each of the polymerases. All the mutants maintain the high accuracy associated with wild type Pfu-Pol and Tkod-Pol. Although Tkod-Pol has a measurably higher exonuclease activity than all the Pfu-Pol derivatives (Table 2), this does not translate into higher accuracy as determined using pSJ2. Fidelity critically depends on the balance between exonuclease and polymerase rates and with Tkod-Pol both are elevated compared with PfuPol. The ratio of polymerase/exonuclease activities may be similar for both enzymes, leading to equal propensity to continue synthesis vs. engaging proof-reading and conferring similar overall fidelity on both proteins.

\section{PRIMER-TEMPLATE BINDING}

The influence of the mutations on primer-template binding was determined using fluorescence anisotropy with hexachlorofluorescein-labeled primer-templates. Previous studies, carried out at pH 7.5 and $100 \mathrm{mM} \mathrm{NaCl}$ (ionic strength $=100$ ), indicated relatively poor binding of Pfu-Pol to primer-templates, a $K_{D}$ of $270 \mathrm{nM}$ being measured (Shuttleworth et al., 2004). As expected, binding affinity was increased at the lower salt concentration of $20 \mathrm{mM} \mathrm{KCl}$ (ionic strength $=20$ ), reflected in a $K_{D}$ of $32 \mathrm{nM}$ (Richardson et al., 2013). In this publication binding titrations were performed in the same buffer used for extensions; $\mathrm{pH} 8.5$ containing $10 \mathrm{mM} \mathrm{KCl}$ and $10 \mathrm{mM}\left(\mathrm{NH}_{4}\right)_{2} \mathrm{SO}_{4}$ (ionic strength of 40). Under these conditions wild type Pfu-Pol bound the primertemplate with a $K_{D}$ of $251 \mathrm{nM}$ (supplementary data, Figure S6). Tkod-Pol showed similar affinity $\left(K_{D}=276 \mathrm{nM}\right)$ and so the more rapid primer-template extensions seen with this enzyme, cannot simply be accounted for by tighter binding to the DNA substrate. The two double mutants showed better interaction with DNA, by a factor a little greater than two for L381R/K502R and just over three for M247R/L381R (supplementary data, Figure S6). Surprisingly Pfu-KodTS bound primer-template $\left(K_{D}=95 \mathrm{nM}\right)$ more strongly than either of the parent polymerases from which it is derived and a further improvement was observed with the Pfu-KodTS L381R/K502R $\left(K_{D}=50 \mathrm{nM}\right)$. All The $K_{D}$ values seen are summarized in Table 2.

\section{PROCESSIVITY OF DNA POLYMERASES}

The processivity of a polymerase is the number of dNTPs incorporated per binding event (Von Hippel et al., 1994; Bambara et al., 
A

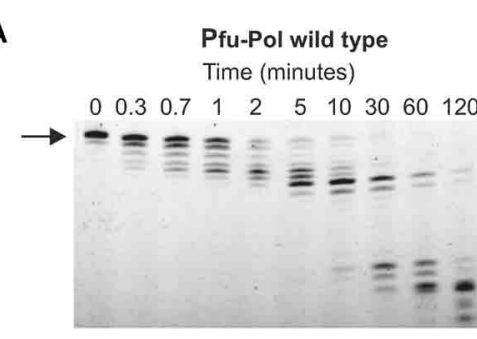

B

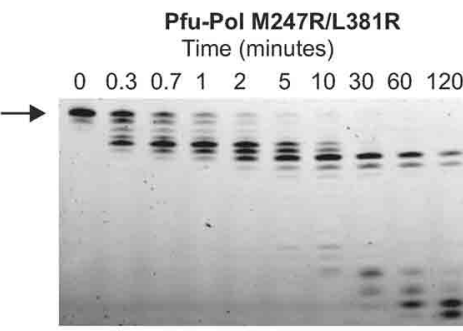

C

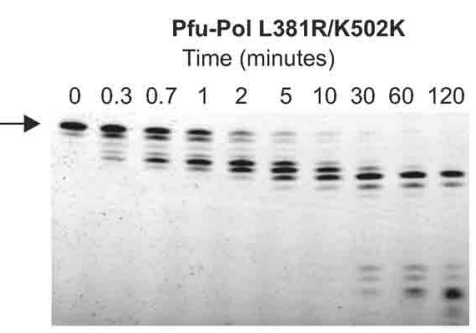

G

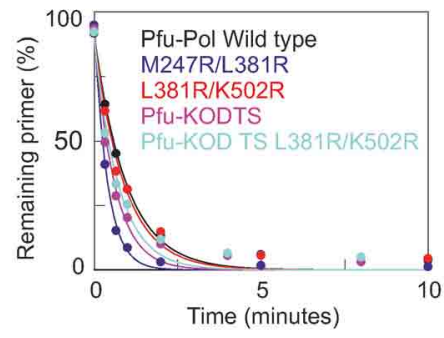

FIGURE 6 | Proof reading $3^{\prime}-5^{\prime}$ exonuclease activity by archaeal family-B DNA polymerases. (A-F) Gel electrophoresis analysis of primer strand degradation seen with a base mismatch primer-template using the polymerase variants indicated. The starting primer is shown arrowed. Panels (G) and $\mathbf{( H )}$ show fits of the data (as \% primer remaining with time) to a single

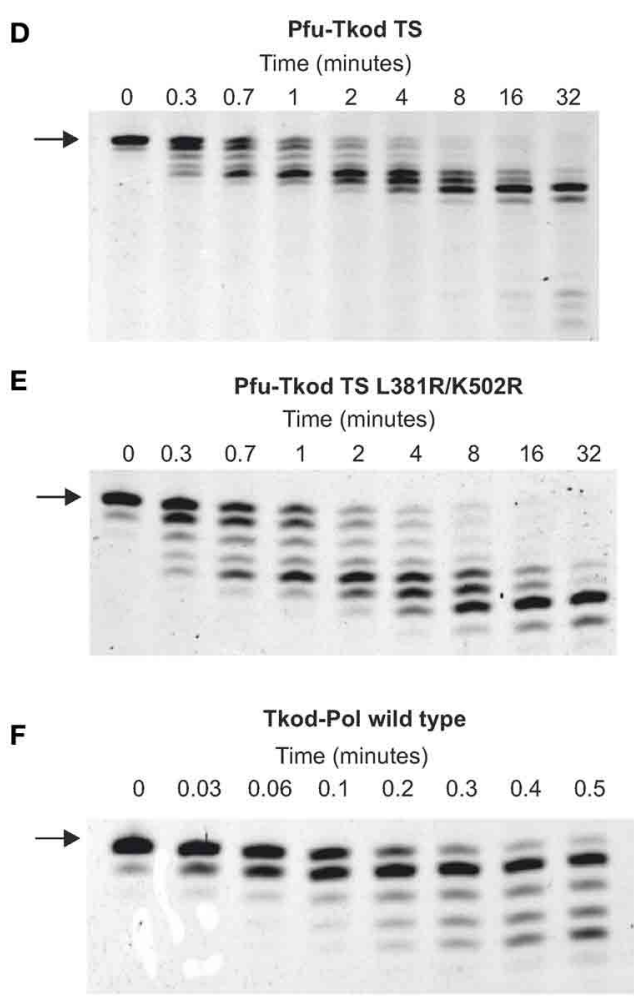

H

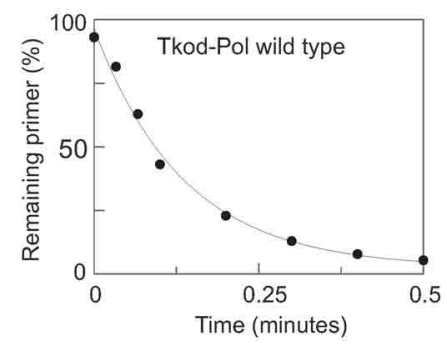

exponential decay to give the rate constants summarized in Table 2. The primer-template used was: 5'-GGGGATCCTCTAGAGTCGACCTGC 3'-CCCCTAGGAGATCTCAGCTGGACAACCGTTCGTTCGAACAGAGG. The primer was labeled at its $5^{\prime}$-terminus with fluorescein. The mismatched bases are shown underlined,
1995).For accurate measurement each primer should undergo only one round of extension and, under these single hit conditions, the number of dNTPs incorporated equals the processivity. Single hits can be achieved using a low concentration of polymerase, relative to primer-template, such that the probability of secondary initiation is low. Alternatively a trap, such as heparin or poly(dA-dT), can be used to sequester the polymerase after it dissociates from the primer-template (Bambara et al., 1995). Archaeal DNA polymerases bind tightly to uracilcontaining DNA (Shuttleworth et al., 2004), enabling a 23-mer containing 5 uracil residues (Figure 7) to be used as trap. The uracil-rich trap bound all polymerase variants with $K_{D}$ values in the $1-10 \mathrm{nM}$ range (data not shown). For processivity determination $40 \mathrm{nM}$ primer-template (sequence given in the legend to Figure 7) and $500 \mathrm{nM}$ polymerase were pre-incubated in the absence of $\mathrm{Mg}^{2+}$ and reactions initiated by the simultaneous addition of the metal and $10 \mu \mathrm{M}$ of the trap. These stringent conditions, a 250-fold excess of a uracil-rich oligodeoxynucleotide with a high affinity for polymerase, should ensure efficient polymerase sequestration and inhibit re-binding to primer-template.

All polymerases were observed to have very low processivities at 250-fold trap excess and nearly identical results were see with a 100 -fold excess (data not shown). Pfu-Pol acted largely in a distributive manner, with little product seen at short time intervals (Figure 7A; larger versions of all processivity gels are given in 
Table 2 | Summary of the properties of the polymerases investigated.

\begin{tabular}{|c|c|c|c|c|c|}
\hline DNA-Pol & $K_{D}(\mathrm{nM})^{\mathrm{a}}$ & $\begin{array}{c}k_{\text {exo }}\left(\min ^{-1}\right) \\
(\text { mis-paired DNA })^{b}\end{array}$ & $\begin{array}{c}k_{\text {exo }}\left(\min ^{-1}\right) \\
(\text { base-paired DNA })^{c}\end{array}$ & Processivity $^{d}$ & $\begin{array}{l}\text { Melting temperature } \\
\qquad\left({ }^{\circ} \mathrm{C}\right)^{\mathrm{e}}\end{array}$ \\
\hline Pfu-Pol wild type & $251 \pm 23$ & $1.0 \pm 0.1$ & $0.17 \pm 0.02$ & 1 & $93.2 \pm 0.5$ \\
\hline Pfu-Pol L381R/K502R & $107 \pm 13$ & $1.2 \pm 0.1$ & nd & $1-3$ & $94.5 \pm 0.8$ \\
\hline Pfu-TkodTS & $95 \pm 7$ & $1.8 \pm 0.1$ & nd & $1-3$ & $95.3 \pm 1.0$ \\
\hline Pfu-TkodTS L381R/K502R & $50 \pm 7$ & $1.7 \pm 0.1$ & nd & 3 & $95.3 \pm 0.6$ \\
\hline
\end{tabular}

${ }^{a}$ The affinity for DNA measured using fluorescence anisotropy titrations (supplementary data, Figure S6). Each value is the average of three determinations \pm the standard deviation. The primer-template used had the following sequence (Hex = hexachlorofluorescein): 5'-HexGGGGATCCTCTAGAGTCGACCTGC $3^{\prime}$ CCCCTAGGAGATCTCAGCTGGACGACCGTTCGTTCGAACAGAGG. (the mismatched bases are underlined.)

${ }^{b}$ Rate observed for the degradation of a mis-paired primer-template, determined with polymerase in excess of DNA (Figure 6). Each value is the average of three determinations \pm the standard deviation. For the mis-paired DNA, the same sequence as given above was used with the underlined dG changed to dA, giving a $d C: d A$ mis-match at the primer-template junction. Fluorescein (Flu) was used as indicator dye.

${ }^{c}$ Rate observed for the degradation of a fully base-paired primer-template, determined with polymerase in excess of DNA (supplementary data, Figure S5). Each value is the average of three determinations \pm the standard deviation. This primer-template has the exact sequence given above and fluorescein (Flu) was used as indicator dye.

${ }^{d}$ Processity (number of dNTPs incorporated per binding event) of the polymerases measured using a uracil-containing single stranded DNA trap (Figure 7).

e Melting temperatures were determined by DSF in the presence of $2 \mathrm{M}$ guanidinium hydrochloride (Figure 8). The $T_{m}$ of the first transition observed is given as an average \pm standard deviation from three measurements.

Table 3 | Fidelities of DNA polymerases determined using the lac $Z \alpha$ indicator pSJ2.

\begin{tabular}{lcccc}
\hline DNA-Pol & $\begin{array}{c}\text { Total } \\
\text { colonies }^{\mathbf{a}}\end{array}$ & $\begin{array}{c}\text { Mutant } \\
\text { (white) } \\
\text { colonies }\end{array}$ & $\begin{array}{c}\text { Mutation } \\
\text { frequency } \\
\text { (corrected) }\end{array}$ & Error rate $^{\mathbf{b}}$ \\
\hline Pfu-Pol wild typed & 25,700 & 11 & $3.2 \times 10^{-4}$ & $1.6 \times 10^{-6}$ \\
Pfu-Pol M247R/L381R & 20,555 & 8 & $2.8 \times 10^{-4}$ & $1.4 \times 10^{-6}$ \\
Pfu-Pol L381R/K502R & 26,675 & 9 & $2.3 \times 10^{-4}$ & $1.2 \times 10^{-6}$ \\
Pfu-TkodTS & 39,814 & 16 & $2.9 \times 10^{-4}$ & $1.5 \times 10^{-6}$ \\
Pfu-TkodTSL381R/K502R & 31,704 & 12 & $2.7 \times 10^{-4}$ & $1.3 \times 10^{-6}$ \\
Tkod-Pol wild type & 28,028 & 11 & $2.8 \times 10^{-4}$ & $1.4 \times 10^{-6}$ \\
\hline
\end{tabular}

a Sum of three independent experiments, each consisting of five repeats.

${ }^{b}$ The Mutation frequency is the ratio mutant (white) colonies/total colonies and has been corrected by subtracting the background mutation frequency of $1.1 \times$ $10^{-4}$ found for gapped pSJ2 (Keith et al., 2013).

$c$ The Error rate is the number of mistakes made by the polymerase per base incorporated. The determination of the error rate from the mutation frequency has been described previously (Keith et al., 2013).

${ }^{d}$ Data taken from an earlier publication (Keith et al., 2013).

the supplementary data, Figure S7). A very faint band representing a single dNTP addition is seen after 2 min and +1 and +2 bands are visible after $5 \mathrm{~min}$. Longer products were observed at 15 and $30 \mathrm{~min}$ but may result from multiple binding events. Thus the uracil trap is not perfect and at longer times polymerase "escape" is seen, maybe arising from trap degradation by the exonuclease activity. As expected, polymerization was more extensive when the trap was omitted (Figure 7A). Adding the trap in the pre-incubation step results in the abolition of the barely extended bands seen at short times; instead only slower mobility bands are observed after prolonged incubation, which correspond well with the similar bands observed when the uracil trap is used in the standard manner. Pfu-Pol has a processivity of 1 (values are summarized in Table 2), implying dissociation prior to adding even a single dNTP is highly likely. With Tkod-Pol a prominent band, corresponding to the incorporation of 3 dNTPs is seen at the shortest time $(5 s)$ in the presence of the trap, representing the processivity (Figure 7E, Table 2). Tkod-Pol is more active than Pfu-Pol and even in the presence of trap full length product, presumably due to multiple binding events, was seen at short times. As expected these bands were more prominent when the trap was left out. Pronounced exonuclease activity, evidenced by shortened primer products was also apparent. The four mutants showed processivity profiles near those of the wild types (Figures 7B-D, Table 2). The double mutant M247A/L381R was very similar to Pfu-Pol with a faint band, representing incorporation of a single dNTP just visible at the one minute time point. L381R/K502R appeared marginally better, the band indicating one dNTP addition was predominant at times of 1 and $2 \mathrm{~min}$, but fainter bands corresponding to the +2 and +3 products were seen. The processivity of this mutant, 1-3, may be slightly improved. Processivity does not need to be a single integral value; rather polymerases show a spread of dNTP incorporations per binding event. Pfu$\operatorname{Kod}(\mathrm{TS})$ also demonstrated a processivity of between 1 and 3 (Figure 7C). Finally Pfu-TkodTSL381R/K502R appeared to be equivalent to Tkod-Pol with a single band at the +3 location visible at short times. Overall Tkod-Pol has higher processivity than Pfu-Pol (3 vs. 1) and with the mutations values are shifted toward Tkod-Pol.

The processivities determined here, using a uracil-rich oligodeoxynucleotide trap, are much lower than previously observed. Values of 270 and $>300$ have been reported for TkodPol and of 6, <20 and 80 for Pfu-Pol (Takagi et al., 1997; Wang et al., 2004; Kim et al., 2007). None of these studies used a trap to hinder re-binding of the polymerase and secondary extension 


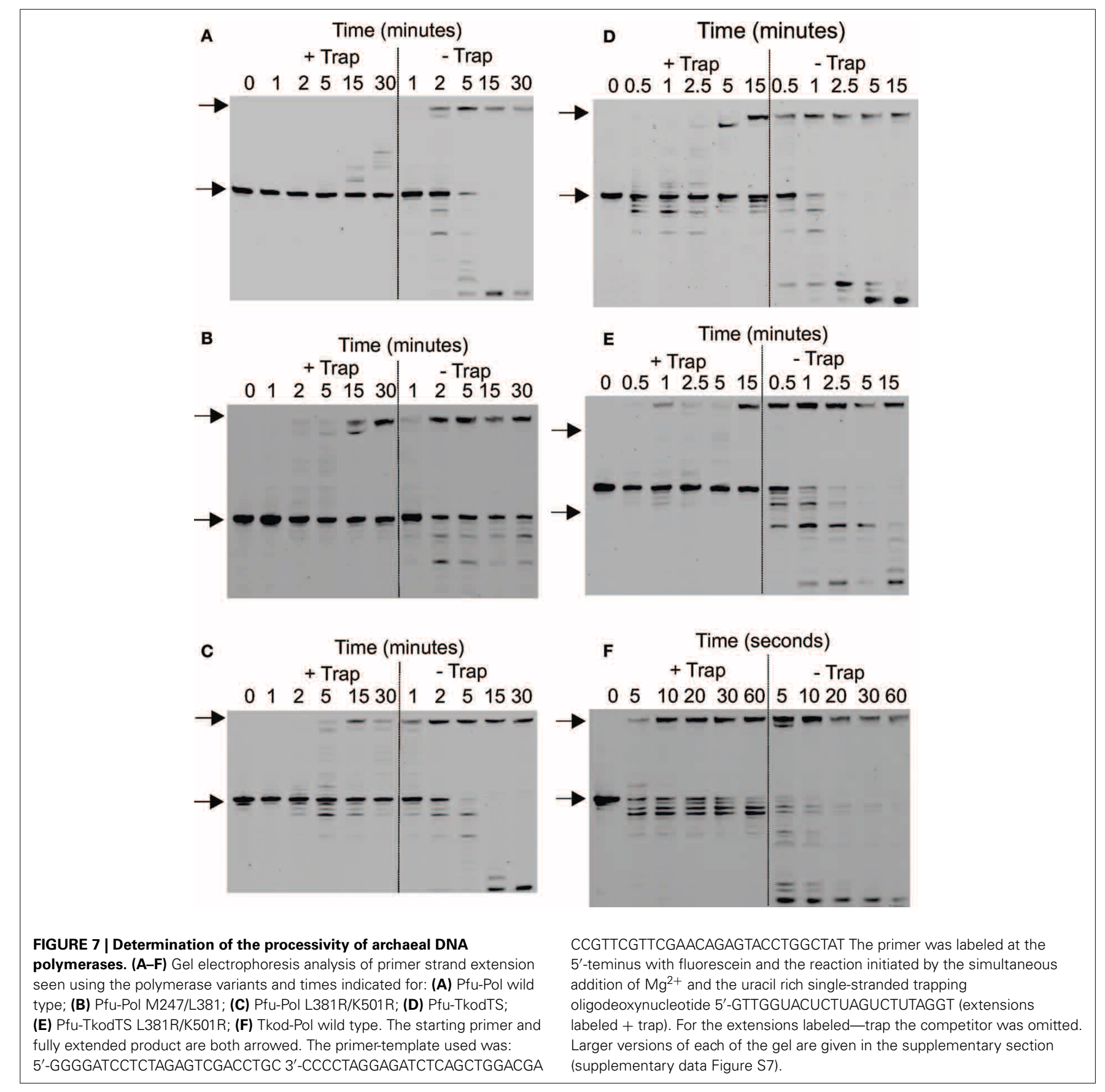

and the change in protocol may explain the discrepancy. All studies agree, however, that Tkod-Pol is more processive than Pfu-Pol. Yeast DNA polymerase $\delta$, similar in structure to the archaeal enzymes (Swan et al., 2009), shows a processivity of 2-3 when evaluated using a trap (Hogg et al., 2014).

\section{THERMOSTABILITY OF DNA POLYMERASES}

The ability to survive elevated temperature is an essential polymerase feature for the PCR. Both archaea, from which the polymerases used in this study are isolated, are hyper-thermophiles, but their preferred growth temperatures differ. Pyrococcus furiosus grows optimally at $100^{\circ} \mathrm{C}$ and can survive at $110^{\circ} \mathrm{C}$, whereas Thermococcus kodakarensis grows best at $85^{\circ} \mathrm{C}$ and can tolerate $94^{\circ} \mathrm{C}$ (Fiala and Stetter, 1986; Borges et al., 2010). Our group earlier measured the thermostability of Pfu-Pol using differential scanning fluorimetry (DSF), where the protein is subject to a steady increase in temperature in the presence of the dye SYPRO orange (Killelea and Connolly, 2011). As thermally-induced unfolding takes place the dye binds to exposed hydrophobic regions, resulting in an increase in fluorescence that can be measured using a real time PCR apparatus. Pfu-Pol was found to be extremely thermostable with the unfolding transition being 
incomplete at $100^{\circ} \mathrm{C}$, the maximum achievable with this technique. Addition of guanidinium hydrochloride $(\mathrm{GuHCl})$ destabilizes Pfu-Pol, making thermal unfolding more accessible. A Pfu-Pol mutant, that lacked two disulphide bridges and was more heat sensitive, demonstrated two well separated melting transitions. Most likely the wild type behaves similarly but the second transition cannot be observed as it takes place above $100^{\circ} \mathrm{C}$ (Killelea and Connolly, 2011). In the present study $2 \mathrm{M} \mathrm{GuHCl}$ was used to bring heat-induced unfolding into the DSF range and the melting profiles are shown in Figure 8. Tkod-Pol shows two melting transitions, the first of which has a $T_{m}$ of $82.9^{\circ} \mathrm{C}$ (all $T_{m}$ values are summarized in Table 2). Pfu-Pol is more stable with a $T_{m}$ of $93.2^{\circ} \mathrm{C}$. As postulated earlier it is probable that a second melting event for Pfu-Pol takes place above $100^{\circ} \mathrm{C}$; therefore, only the first observed transitions have been used for comparing unfolding. The $T_{m}$ of $93.2^{\circ} \mathrm{C}$ is slightly higher than that of $89.7^{\circ} \mathrm{C}$ observed earlier for Pfu-Pol in $2 \mathrm{M} \mathrm{GuHCl}$ (Killelea and Connolly, 2011). Such variation may be accounted for by the different $\mathrm{NaCl}$ concentrations used, $400 \mathrm{mM}$ here, $200 \mathrm{mM}$ previously. Unsurprisingly the double mutants of Pfu-Pol, M247R/L381R $\left(T_{m}=95.7^{\circ} \mathrm{C}\right.$ and $\mathrm{L} 381 \mathrm{R} / \mathrm{K} 502 \mathrm{R}\left(T_{m}=94.5^{\circ} \mathrm{C}\right)$ retain the thermostability of the wild type, if anything being slightly more heat resistant. More unexpectedly the thumb swaps, in which about $24 \%$ of the protein is derived from Tkod-Pol, fully retain the stability of Pfu-Pol, with no lowering of $T_{m}$, toward that of Tkod-Pol is (Figure 8 and Table 2).

\section{DISCUSSION}

The use of the PCR across biological, medical, veterinary, agricultural and forensic sciences has aroused considerable interest

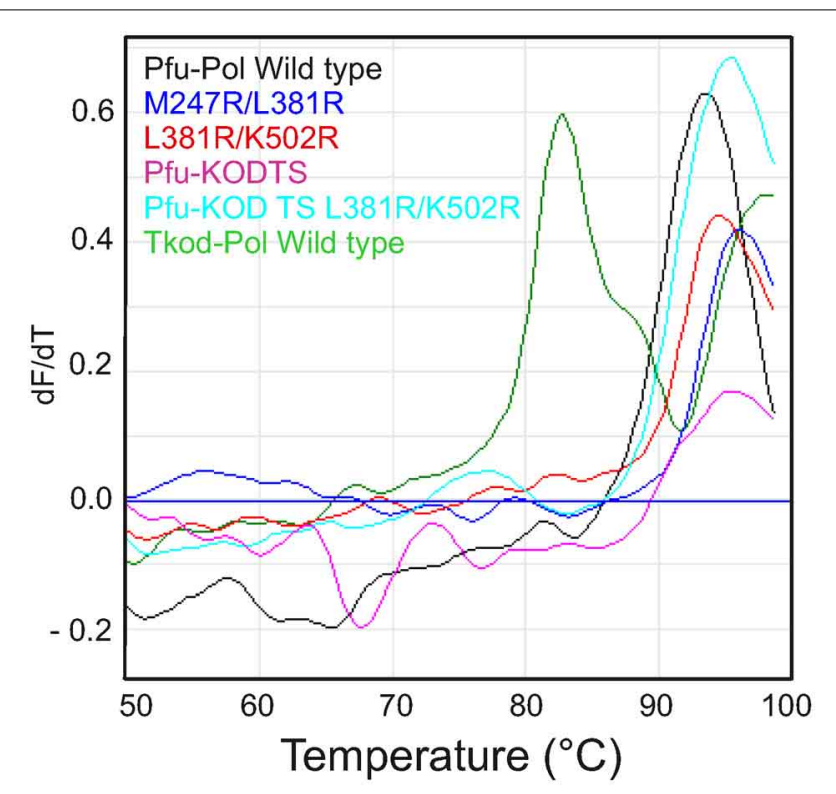

FIGURE 8 | Differential scanning fluorimetry (DSF) profiles describing the thermal unfolding of the polymerases. The first derivatives of the DSF profiles are shown with $d F / d T$ indicating the change in fluorescence (relative units). Individual polymerases are identified by the color coding given in the figures. in thermostable DNA polymerases and many enzymes, especially from the Thermococcales order of the archaea, are employed (Terpe, 2013). Tkod-Pol and Pfu-Pol are the most established and several investigations have pointed out the advantageous nature of the former in terms of speed and processivity (Takagi et al., 1997; Nishioka et al., 2001; Kim et al., 2007). The abundance of arginines at the "forked-point" has been offered as one reason for the high processivity of Tkod-Pol (Hashimoto et al., 2001; Kim et al., 2008), but the absence of a closed ternary complex (enzyme/primer-template/dNTP) limits knowledge on the exact functions of these amino acids. It was originally proposed that Tkod-Pol R247 may separate the primer-template and stabilize the denatured substrate, in a similar manner to corresponding amino acid in the bacteriophage RB69 polymerase (Shamoo and Steitz, 1999; Hashimoto et al., 2001). More recent studies have suggested that this amino acid is relatively unimportant (Aller et al., 2011; Richardson et al., 2013). The thumb domain of archaeal family-B polymerases, and indeed all DNA polymerases, is responsible for binding double-stranded DNA and expected to be critical in DNA translocation and processivity. Several "forkedpoint" arginines are missing in Pfu-Pol and the thumb domain shows subtle differences between the two enzymes. A major aim of this study was to graft these elements into Pfu-Pol in the hope of creating better PCR enzymes.

As "forked-point" arginines and/or the thumb domain of Tkod-Pol are introduced into Pfu-Pol, extension rates become faster, PCR performance improves and processivity, albeit only from one to three, is increased. In all cases the superior thermostability of Pfu-Pol is retained and there is no decrease in fidelity. The influence appears cumulative with the best mutant, combining both "forked-point" and thumb alterations. PfuTkodTS L381R/K502R represents an end-point (the entire thumb has been exchanged and addition of further missing arginines e.g., R246 does not enhance properties), yet still falls short of the performance of Tkod-Pol. This suggests other regions of the polymerase may play a role and these may subtly differ between Tkod-Pol and Pfu-Pol. One important area is the fingers domain, made up of two long $\alpha$-helices (Hopfner et al., 1999; ChapinRodriguez et al., 2000; Hashimoto et al., 2001; Kim et al., 2008), which undergoes a conformational change following dNTP binding to the polymerase/primer-template complex, to produce a catalytically competent "closed" ternary complex. The fingers domain is highly conserved in all DNA polymerases and plays a critical role in $\mathrm{dNTP}$ selection and accurate insertion of the incoming base into the extending primer (Brautigam and Steitz, 1998). The amino acid sequences of the fingers domain of TkodPol and Pfu-Pol are similar, but not identical (supplementary data, Figure S1). Given the critical function of this area in catalysis, the small variations may impinge on PCR performance. A second key element may be the Y-GG/A motif (Brautigam and Steitz, 1998), shown to be important in the processivity, fidelity and PCR capability of archaeal polymerases (Bohlke et al., 2000). The sequences near this region are shown in Figure 1, corresponding to the amino acids near arginine 381 (Y-GG/A = YEGG for Tkod-Pol and YTGG for Pfu-Pol). The presence of an additional leucine (L381) in Pfu-Pol alters the location of the conserved tyrosine and glycines, moving them away from 
the DNA phosphate backbone and disrupting conserved proteinDNA interactions (Kim et al., 2008). Deletion of this leucine did result in slightly improved primer-template extension, but it is not clear if this mutation correctly lines up the subsequent arginine in Pfu-Pol with R381 of Tgo-Pol (Figure 1) and the L381R substitution was slightly superior (supplementary data, Figure S2). Of course Pfu-PolL381R still retains an extra amino acid and this may disrupt the downstream Y-GG/A motif as seen for the wild type. Finally communication between the exonuclease and thumb domains has been implicated in the co-ordination of polymerase and proof-reading exonuclease activities (Kuroita et al., 2005; Kim et al., 2008). Trans-domain interactions between amino acids in these regions appear to control the relative motions of the two domains, influencing catalytic activities. The inter-domain contacts appear to vary slightly between Tkod-Pol and Pfu-Pol, potentially impinging on PCR properties. Such considerations may be especially significant with the thumb swap mutants.

The significance of this publication is the demonstration that it is relatively simple to improve the PCR performance of Pfu-Pol by substituting individual amino acids and even an entire domain found in the closely related Tkod-Pol. The Pfu-Pol framework seems remarkably tolerant to substantial alteration, maintaining thermostability and fidelity. Future possibilities include incorporating elements from other Thermococcales family-B polymerases; a reasonable number are known (Figure 1) and many have been applied in the PCR (Terpe, 2013). Further improvement may be achieved by combining gene segments using random techniques such as DNA shuffling (Stemmer, 1994) or staggered extension process (StEP) (Zhao et al., 1998) and selecting for improved PCR performance with compartmentalized selfreplication (CSR) (Ghadessy and Holliger, 2007). This approach may even be extending to environmental DNA from uncharacterized archaea, widening the genetic resource (Matsukawa et al., 2009). Experiments could target the "forked-point" and thumb, as well as other elements, suggested above to play a role in PCR ability. Such an undertaking should yield important clues about features of archaeal DNA polymerases that are important for efficient and robust PCR and may, ultimately, lead to superior reagents. It may be possible to develop DNA polymerases more suited to demanding PCR applications such as amplifying DNA from single cells or old/damaged/degraded sources and in the steps needed prior to high throughput DNA sequencing.

\section{AUTHOR CONTRIBUTIONS}

All authors carried out experiments to acquire data and participated in analysing and interpreting the results. Bernard A. Connolly originally conceived the project and Ashraf M. Elshawadfy, Brian J. Keith, and Thomas Kinsman contributed to experimental design. Bernard A. Connolly wrote the first draft of the work and all authors contributed in critical revision. All authors concur with the final version and agree to take responsibility for the work and conclusions described within.

\section{ACKNOWLEDGMENTS}

Ashraf M. Elshawadfy was supported by grants from the Egyptian government and the University of Zagazig (Zagazig, Egypt). Brian J. Keith and Thomas Kinsman were UK BBSRC-supported Ph.D. students. The research was supported by a Wellcome Trust (UK) equipment grant (grant number 064345). Zvi Kelman is thanked for kindly supplying the Tkod-Pol B overexpressing plasmid.

\section{SUPPLEMENTARY MATERIAL}

The Supplementary Material for this article can be found online at: http://www.frontiersin.org/journal/10.3389/fmicb. 2014.00224/abstract

\section{REFERENCES}

Aller, P., Duclos, S., Wallace, S. S., and Doublié, S. (2011). A crystallographic study of the role of sequence context in thymine glycol bypass by a replicative DNA polymerase serendipitously sheds light on the exonuclease complex. J. Mol. Biol. 412, 22-34. doi: 10.1016/j.jmb.2011.07.007

Bambara, R. A., Fay, P. J., and Mallaber, L. M. (1995). Methods of analysing processivity. Meth. Enzymol. 262, 270-280. doi: 10.1016/0076-6879(95)62023-0

Bergen, K., Betz, W., Welte, W., Diederichs, K., and Marx, A. (2013). Structures of KOD and $9^{\circ} \mathrm{N}$ DNA polymerases complexed with primer template duplex. Chembiochem 14, 1058-1062. doi: 10.1002/cbic.201300175

Bohlke, K., Pisani, F. M., Vorgias, C. F., Frey, B., Sobek, H., Rossi, M., et al. (2000). PCR performance of the B-type DNA polymerase from the thermophilic euryarchaeon Thermococcus aggregans improved by mutations in the Y-GG/A motif. Nucleic Acids Res. 28, 3910-3917. doi: 10.1093/nar/28.20.3910

Borges, N., Matsumi, R., Imanaka, T., Atomi, H., and Santos, H. (2010). Thermococcus kodakarensis mutants deficient in di-myo-inositol phosphate use aspartate to cope with heat stress. J. Bacteriol. 192, 191-197. doi: 10.1128/JB.01115-09

Brautigam, C. A., and Steitz, T. A. (1998). Structural and functional insights provided by crystal structures of DNA polymerases and their substrate complexes. Curr. Opin. Struct. Biol. 8, 54-63. doi: 10.1016/S0959-440X(98)80010-9

Chapin-Rodriguez, A., Park, H.-W., Mao, C., and Beese, L. S. (2000). Crystal structure of a Pol $\alpha$ family DNA polymerase from the hperthermophilic archaeon Thermococcus sp. $9^{\circ}$ N-7. J. Mol. Biol. 299, 447-462. doi: 10.1006/jmbi.20 00.3728

Cline, J., Braman, J. C., and Hogrefe, H. H. (1996). PCR fidelity of Pfu DNA polymerase and other thermostable DNA polymerases. Nucleic Acids Res. 18, 3546-3551. doi: 10.1093/nar/24.18.3546

Emptage, K., O’Neill, R., Solovyova, A., and Connolly, B. A. (2008). Interplay between DNA polymerase and proliferating cell nuclear antigen switches off base excision repair of uracil and hypoxanthine during replication in archaea. J. Mol. Biol. 383, 762-771. doi: 10.1016/j.jmb.2008.08.018

Evans, S. J., Fogg, M. J., Mamone, A., Davis, M., Pearl, L. H., and Connolly, B. A. (2000). Improving dideoxynucleotide-triphosphate utilization by the hyperthermophilic DNA polymerase from Pyrococcus furiosus. Nucleic Acids Res., 28, 1059-1066. doi: 10.1093/nar/28.5.1059

Fiala, G., and Stetter, K. O. (1986). Pyrococcus furiosus, sp. nov., represents a novel genus of marine heterotrophic archaebacteria growing optimally at $100^{\circ} \mathrm{C}$. Arch. Microbiol. 145, 56-61. doi: 10.1007/BF00413027

Firbank, S. J., Wardle, J., Heslop, P., Lewis, R. J., and Connolly, B. A. (2008). Uracil recognition in archaeal DNA polymerases captured by X-ray crystallography. J. Mol. Biol. 381, 529-539. doi: 10.1016/j.jmb.2008.06.004

Ghadessy, F. J., and Holliger, P. (2007). Compartmentalized self-replication: a novel method for the directed evolution of polymerases and other enzymes. Methods Mol. Biol. 352, 237-248.

Gougel, J., Ralec, C., Henneke, G., and Delarue, M. (2012). Molecular recognition of canonical and deaminated bases by $P$. abyssi family B DNA polymerase. J. Mol. Biol. 423, 315-336. doi: 10.1016/j.jmb.2012.07.025

Hashimoto, H., Nishioka, M., Fujiwara, S., Takagi, M., Imanaka, T., Inoue, T., et al. (2001). Crystal structure of DNA polymerase from hyperthermophilic archaeon Pyrococcus kodakaraensis KOD1. J. Mol. Biol. 306, 469-477. doi: 10.1006/jmbi.2000.4403

Hogg, M., Ostermal, P., Byundl, G. O., Ganail, R. A., Lundström, E.-B., SauerEriksson, A. E., et al. (2014). Structural basis for processive DNA synthesis by yeast DNA polymerase $\varepsilon$. Nat. Struct. Mol. Biol. 21, 49-55. doi: 10.1038/nsmb. 2712

Hopfner, K. P., Eichinger, A., Engh, R. A., Laue, F., Ankenbauer, W., Huber, R., et al. (1999). Crystal structure of a thermostable type B DNA polymerase from 
Thermococcus gorgonarius. Proc. Natl. Acad. Sci. U.S.A. 96, 3600-3605. doi: 10.1073/pnas.96.7.3600

Ishino, S., Kawamura, A., and Ishino, Y. (2012). Application of PCNA to processive PCR by reducing the stability of its ring structure. J. Jpn. Soc. Extremophiles 11, 19-25.

Ishino, Y., and Ishino, S. (2011). Rapid progress of DNA replication studies in Archaea, the third domain of life. China Life Sci. 55, 386-403. doi: 10.1007/s11427-012-4324-9

Jozwiakowski, S. J., and Connolly, B. A. (2009). Plasmid-based lacZ $\alpha$ assay for DNA polymerase fidelity: application to archaeal family-B DNA polymerase. Nucleic Acids Res. 37, e102. doi: 10.1093/nar/gkp494

Keith, B. J., Jozwiakowski, S. K., and Connolly, B. A. (2013). A plasmid-based lacZ $\alpha$ gene assay for DNA polymerase fidelity measurement. Anal. Biochem. 433, 153-161. doi: 10.1016/j.ab.2012.10.019

Killelea, T., and Connolly, B. A. (2011). Role of disulfide bridges in archaeal family-B DNA polymerases. Chembiochem 12, 1330-1336. doi: 10.1002/cbic. 201100145

Killelea, T., Ghosh, S., Tan, S. S., Heslop, P., Firbank, S., Kool, E. T., et al. (2010). Probing the interaction of archaeal DNA polymerases with deaminated bases using X-ray crystallography and non-hydrogen bonding isosteric base analogues. Biochemistry 49, 5772-5781. doi: 10.1021/bi100421r

Kim, S. W., Kim, D.-U., Kim, J. K., Kang, L.-W., and Cho, H.-S. (2008). Crystal structure of $P f u$, the high fidelity DNA polymerase from Pyrococcus furiosus. Int. J. Biol. Macromol. 42, 356-361. doi: 10.1016/j.ijbiomac.2008.01.010

Kim, Y. J., Lee, H. S., Bae, S. S., Jeon, J. H., Lim, J. K., Cho, Y., et al. (2007). Cloning, purification, and characterization of a new DNA polymerase from a hyperthermophilic archaeon, Thermococcus sp. NA1. J. Microbiol. Biotechnol. 17, 1090-1097.

Kitabayashi, M., Nishiya, Y., Esaka, M., Itakura, M., and Imanaka, T. (2002). Gene cloning and polymerase chain reaction with proliferating cell nuclear antigen from Thermococcus kodakaraensis KOD1. Biosci. Biotechnol. Biochem. 66, 2194-2200. doi: 10.1271/bbb.66.2194

Kuroita, T., Matsumura, H., Yokota, N., Kitabayashi, M., Hashimoto, H., Inoue, T., et al. (2005). Structural mechanism for coordination of proofreading and polymerase activities in archaeal DNA polymerases. J. Mol. Biol. 351, 291-298. doi: 10.1016/j.jmb.2005.06.015

Lundberg, K. S., Shoemaker, D. D., Adams, M. W. W., Short, J. M., and Mathur, E. J. (1991). High-fidelity amplification using a thermostable DNA polymerase isolated from Pyrococcus furiosus. Gene 108, 1-6. doi: 10.1016/03781119(91)90480-Y

Matsukawa, H., Yamagami, T., Kawarabayasi, Y., Miyashita, Y., Takahashi, M., and Ishino, Y. (2009). A useful strategy to construct DNA polymerases with different properties by using genetic resources from environmental DNA. Genes Genet. Syst. 84, 3-13. doi: 10.1266/ggs.84.3

McPherson, M. J., and Möller, S. G. (2006). PCR. Boca Raton, FL: Taylor and Francis.

Mullis, K. B., Ferre, F., and Gibbs, R. A. (1994). The Polymerase Chain Reaction (PCR). Basel: Birkhäuser Verlag AG. doi: 10.1007/978-1-4612-0257-8

Nishioka, M., Mizuguchi, H., Fujiwara, S., Komatsubara, S., Kitabayashi, M., Uemura, H., et al. (2001). Long and accurate PCR with a mixture of KOD DNA polymerase and its exonuclease deficient mutant enzyme. J. Biotech. 88, 144-149. doi: 10.1016/S0168-1656(01)00275-9

Pavlov, A. R., Belova, G. I., Kozyavkin, S. A., and Slesarev, A. I. (2002). Helix-turn-helix motifs confer salt resistance and processivity on chimeric DNA polymerases. Proc. Natl. Acad. Sci. U.S.A. 99, 13510-13515. doi: 10.1073/pnas.202127199

Reha-Krantz, L. J. (2010). DNA polymerase proofreading: Multiple roles maintain genome stability. Biochimica Biophysica Acta 1804, 1049-1063. doi: 10.1016/j.bbapap.2009.06.012

Richardson, T. T., Wu, X., Keith, B. J., Heslop, P., Jones, A. C., and Connolly, B. A. (2013). Unwinding of primer-templates by archaeal family-B DNA polymerases in response to template-strand uracil. Nucleic Acids Res. 41, 2466-2478. doi: $10.1093 /$ nar/gks1364
Russell, H. J., Richardson, T. T., Emptage, K., and Connolly, B. A. (2009). The $3^{\prime}-$ $5^{\prime}$ proofreading exonuclease of archaeal family-B DNA polymerase hinders the copying of template strand deaminated bases. Nucleic Acids Res. 37, 7603-7611. doi: 10.1093/nar/gkp800

Saunders, N. A., and Lee, M. (2013). Real Time PCR; Advanced Technologies and Applications. Linton: Caister Academic Press.

Shamoo, Y., and Steitz, T. A. (1999). Building a replisome from interacting pieces: sliding clamp complexed to a peptide from DNA polymerase and a polymerase editing complex. Cell 99, 155-166. doi: 10.1016/S0092-8674(00)81647-5

Shuttleworth, G., Fogg, M. J., Kurpiewski, M. R., Jen-Jacobson, L., and Connolly, B. A. (2004). Recognition of the pro-mutagenic base uracil by family B DNA polymerases from archaea. J. Mol. Biol. 337, 621-634. doi: 10.1016/j.jmb.2004.01.021

Sievers, F., Wilm, A., Dineen, D., Gibson, T. J., Karplus, K., Li, W., et al. (2011). Fast, scalable generation of high-quality protein multiple sequence alignments using Clustal Omega. Mol. Syst. Biol. 7:539. doi:10.1038/msb.2011.75

Stemmer, C. P. (1994). Rapid evolution of a protein in vitro by DNA shuffling. Nature 370, 389-391. doi: 10.1038/370389a0

Swan, M. K., Johnson, R. E., Prakash, L., Prakash, S., and Aggarwal, A. K. (2009). Structural basis of high-fidelity DNA synthesis by yeast DNA polymerase $\delta$. Nat. Struct. Mol. Biol. 16, 979-986. doi: 10.1038/nsmb.1663

Takagi, M., Nishioka, M., Kakihara, H., Kitabayashi, M., Inoue, H., Kawakami, B., et al. (1997). Characterization of DNA polymerase from Pyrococcus sp. strain KOD1 and its application to PCR. Appl. Environ. Microbiol. 63, 4504-4510.

Terpe, K. (2013). Overview of thermostable DNA polymerases for classical PCR applications: from molecular and biochemical fundamentals to commercial systems. Appl. Microbiol. Biotechnol. 97, 10253-10254. doi: 10.1007/s00253-0135290-2

Von Hippel, P. H., Fairfield, F. R., and Dolejsi, M. K. (1994). On the processivity of polymerases. Ann. N.Y. Acad. Sci. 726, 118-131. doi: 10.1111/j.17496632.1994.tb52803.x

Wang, Y., Prosen, D. E., Mei, L., Sullivan, J. C., Finney, M., and Vander Horn, P. B. (2004). A novel strategy to engineer DNA polymerases for enhanced processivity and improved performance in vitro. Nucleic Acids Res. 32, 1197-1207. doi: 10.1093/nar/gkh271

Warrens, A. N., Jones, M. D., and Lechler, R. I. (1997). Splicing by overlap extension by PCR using asymmetric amplification: an improved technique for the generation of hybrid proteins of immunological interest. Gene 186, 29-35. doi: 10.1016/S0378-1119(96)00674-9

Weissensteiner, T., Griffin, H. G., and Griffin, A. M. (2004). PCR Technology: Current Innovations. Boca Raton, FL: CRC Press.

Zhao, H., Giver, L., Shao, Z., Affholter, J. A., and Arnold, F. H. (1998). Molecular evolution by staggered extension process (StEP) in vitro recombination. Nat. Biotechnol. 16, 258-261. doi: 10.1038/nbt0398-258

Conflict of Interest Statement: Brian J. Keith was partially funded by Bioline, a company with commercial interests in PCR enzymes. Apart from this the authors declare that the research was conducted in the absence of any commercial or financial relationships that could be construed as a potential conflict of interest.

Received: 04 April 2014; accepted: 28 April 2014; published online: 27 May 2014. Citation: Elshawadfy AM, Keith BJ, Ee Ooi H, Kinsman T, Heslop P and Connolly $B A$ (2014) DNA polymerase hybrids derived from the family-B enzymes of Pyrococcus furiosus and Thermococcus kodakarensis: improving performance in the polymerase chain reaction. Front. Microbiol. 5:224. doi: 10.3389/fmicb.2014.00224

This article was submitted to Evolutionary and Genomic Microbiology, a section of the journal Frontiers in Microbiology.

Copyright (C) 2014 Elshawadfy, Keith, Ee Ooi, Kinsman, Heslop and Connolly. This is an open-access article distributed under the terms of the Creative Commons Attribution License (CC BY). The use, distribution or reproduction in other forums is permitted, provided the original author(s) or licensor are credited and that the original publication in this journal is cited, in accordance with accepted academic practice. No use, distribution or reproduction is permitted which does not comply with these terms. 